\title{
The environmental dependence of the stellar mass fundamental plane of early-type galaxies
}

\author{
Lei Hou and Yu Wang \\ Department of Astronomy, University of Science and Technology of China, Jinzhai Road 96, 230026 Hefei, PR China \\ e-mail: wywa@ustc.edu.cn
}

Received 23 November 2015 / Accepted 1 February 2016

\section{ABSTRACT}

\begin{abstract}
Aims. We investigate the environmental dependence of the stellar mass fundamental plane $\left(\mathrm{FP}_{*}\right)$ using the early-type galaxy sample from the Sloan Digital Sky Survey data release 7 (SDSS DR7).

Methods. The $\mathrm{FP}_{*}$ is calculated by replacing the luminosity in the FP with stellar mass. Based on the SDSS group catalog, we characterize the galaxy environment according to the mass of the host dark matter halo and the position in the halo. In halos with the same mass bin, the color distributions of central and satellite galaxies are different. Therefore, we calculate $\mathrm{FP}_{*}$ coefficients of galaxies in different environments and compare them with those of the FP to study the contribution of the stellar population.

Results. We find that coefficient $a$ of the $\mathrm{FP}_{*}$ is systematically larger than that of the FP, but coefficient $b$ of the $\mathrm{FP}_{*}$ is similar to the FP. Moreover, the environmental dependence of the $\mathrm{FP}_{*}$ is similar to that of the FP. For central galaxies, $\mathrm{FP}_{*}$ coefficients are significantly dependent on the halo mass. For satellite galaxies, the correlation between $\mathrm{FP}_{*}$ coefficients and the halo mass is weak.

Conclusions. We conclude that the tilt of the FP is not primarily driven by the stellar population.
\end{abstract}

Key words. galaxies: elliptical and lenticular, $\mathrm{cD}$ - galaxies: halos - galaxies: statistics

\section{Introduction}

The fundamental plane (FP) is a scaling relation of early-type galaxies (ETGs) between the central velocity dispersion $\sigma_{0}$, the effective radius $R_{0}$, and the average surface brightness in the effective radius $I_{0}$ (Djorgovski \& Davis 1987; Dressler et al. 1987). It is written as

$\log R_{0}=a \log \sigma_{0}+b \log I_{0}+c$.

The scatter of the FP is quite small. Based on the assumption of structural and dynamical homology, ETGs have similar density and orbital distributions. If ETGs also have similar dark matter fraction and the stellar mass-to-light ratio, and assuming that the virial theorem holds at all radii, a FP with $a=2$ and $b=-1$ is expected. However, observed FP coefficients are found to differ from the virial prediction. This is called the tilt of the FP. Many studies have discussed the origin of the FP tilt, such as the structural and dynamical non-homology (Graham \& Colless 1997; Busarello et al. 1997; Trujillo et al. 2004), the variations of the stellar population (Prugniel \& Simien 1996; Forbes et al. 1998), and the fraction of the dark matter to the total mass (Ciotti et al. 1996; Borriello et al. 2003; Padmanabhan et al. 2004). The conclusions in these studies often conflict with each other and there are also suggestions that the tilt of the FP is due to the contribution of multiple factors (D'Onofrio et al. 2013).

Many works indicate that there is a systematic variation of stellar population parameters through the FP, such as $M_{*} / L$, age, and metallicity (Graves 2009; Springob et al. 2012). Therefore, the stellar population seems to make an important contribution to the FP tilt. To resolve the contribution of the stellar population, Hyde \& Bernardi (2009) measure the stellar mass fundamental plane (hereafter $\mathrm{FP}_{*}$ ) by replacing the luminosity in the $\mathrm{FP}$ with the stellar mass

$\log R_{0}=a \log \sigma_{0}+b \log \Sigma_{0}+c$, where $\Sigma_{0}=M_{*} /\left(2 \pi R_{0}^{2}\right)$ is the stellar mass surface density. The difference between $\mathrm{FP}$ and $\mathrm{FP}_{*}$ is due to the stellar population, and the difference between $\mathrm{FP}_{*}$ and the virial prediction is due to the non-homology and dark matter fraction. As a result, the contribution of stellar population to the FP tilt is resolved. Hyde \& Bernardi (2009) compared the FP and $\mathrm{FP}_{*}$ of ETGs in the Sloan Digital Sky Survey (SDSS), and found that the $\mathrm{FP}_{*}$ is steeper than the FP, but shallower than the virial prediction. This means that the stellar population only contributes to a part of the FP tilt.

The properties of galaxies have been found to correlate with the environment. For example, the propotion of ETGs is larger in denser environment, which is called the morphology-density relation (Dressler 1980). Several studies have focused on the environmental dependence of the FP and found that FP coefficients in denser environments are different to those in lower density environments (Lucey et al. 1991; de Carvalho \& Djorgovski 1992; Bernardi et al. 2003a, 2006; D'Onofrio et al. 2008; La Barbera et al. 2010b; Magoulas et al. 2012; Cappellari et al. 2013b). However, the definitions of the environment in these studies are different and this makes it difficult to compare the conclusions. The SDSS group catalog (Yang et al. 2007) provides an approach for studying the correlation between galaxies and the environment. Each galaxy in the catalog is assigned to a dark matter halo and identified as a central or satellite galaxy in the halo. Based on the group catalog, Hou \& Wang (2015) investigated the environmental dependence of the FP of ETGs in the SDSS, and found that FP coefficients of central galaxies are significantly correlated to the halo mass, but those of satellite galaxies are independent of the halo mass. The discrepancy between central and satellite galaxies is significant in small halos. However, central and satellite galaxies with the same halo mass have different color distribution. The environmental dependence of FP coefficients may be due to the variation of the color. We therefore need to test whether the environmental dependence of FP coefficients 
is due to the variation of the color. The $\mathrm{FP}_{*}$ is the most useful tool to study the effect of the color.

The aim of this paper is to study the environmental dependence of the $\mathrm{FP}_{*}$. To do this, we select the ETG sample from the SDSS data release 7 (DR7), and use the SDSS group catalog to find the halo mass and the position in the halo for each galaxy. Then we measure $\mathrm{FP}_{*}$ coefficients of ETGs in different environments. By comparing the environmental dependence of the FP and the $\mathrm{FP}_{*}$, we can resolve the effect of the stellar population.

The paper is organized as follows. Section 2 describes the formulation of the FP and the $\mathrm{FP}_{*}$. Section 3 introduces the ETG sample, the SDSS group catalog and the calculation of physical parameters. Section 4 describes the fitting method of the $\mathrm{FP}_{*}$. We investigate the environmental dependence of the $\mathrm{FP}_{*}$ in Sect. 5. We summarize this paper and discuss the results in Sect. 6. Throughout this paper, we adopt a $\Lambda$ CDM cosmology with parameter $\Omega_{\mathrm{m}}=0.238, \Omega_{\Lambda}=0.762$ and $h=0.73$.

\section{From the FP to the $\mathrm{FP}_{*}$}

If the virial theorem holds at all radii, ETGs should satisfy

$V^{2} \propto \frac{G M_{\text {dyn }}}{R} \propto \frac{M_{\text {dyn }}}{L} R I$,

where $M_{\text {dyn }}$ is the dynamical mass, $V \equiv \sqrt{v_{c}^{2} / 2+\sigma^{2}}, v_{c}$ is the rotational velocity and $\sigma$ is the velocity dispersion. Assuming $V / \sigma$ is constant for all ETGs, then

$\sigma^{2} \propto \frac{G M_{\text {dyn }}}{R} \propto \frac{M_{\text {dyn }}}{L} R I$.

If $M_{\text {dyn }} / L$ is also constant, the FP with $a=2$ and $b=-1$ is expected. The tilt of the FP is the evidence on the systematic variation of $M_{\mathrm{dyn}} / L$. The dynamical mass-to-light ratio $M_{\mathrm{dyn}} / L$ can be resolved into three factors

$\frac{M_{\mathrm{dyn}}}{L}=\frac{M_{\mathrm{dyn}}}{M_{\mathrm{tot}}} \frac{M_{\mathrm{tot}}}{M_{*}} \frac{M_{*}}{L}$,

where $M_{\text {tot }}$ is the total mass of dark matter and baryonic matter (Hyde \& Bernardi 2009). Each factor corresponds to an explanation to the FP tilt: $M_{\text {dyn }} / M_{\text {tot }}$ represents the non-homology, $M_{\mathrm{tot}} / M_{*}$ represents the fraction of the stellar mass to the total mass, and $M_{*} / L$ represents the stellar population.

To examine the contribution of the stellar population to the FP, Hyde \& Bernardi (2009) replaced $L$ into $M_{*}$ to produce the $\mathrm{FP}_{*}$. Similar to Eq. (4), the virial theorem is expressed as

$\sigma^{2} \propto \frac{G M_{\mathrm{dyn}}}{R} \propto \frac{M_{\mathrm{dyn}}}{M_{*}} R \Sigma$.

If $M_{\mathrm{dyn}} / M_{*}$ is constant, the $\mathrm{FP}_{*}$ satisfies the virial prediction. The effect of stellar population is eliminated from the $\mathrm{FP}_{*}$.

In our work, we calculate the dependence of FP coefficients on the halo mass of central and satellite galaxies. However, in the same halo mass bin, the color distributions of central and satellite galaxies are different. In Fig. 1, we plot $g-r$ color as a function of the halo mass for central and satellite galaxies respectively. We find that central galaxies in more massive halos are redder, but the color of satellite galaxies is only weakly correlated to the halo mass. This means that the environmental dependence of FP coefficients may be caused by the difference of the color distribution between central and satellite galaxies. Therefore, the environmental dependence of the $\mathrm{FP}_{*}$ needs to be calculated, and compared with that of the FP to find the effect of the color.

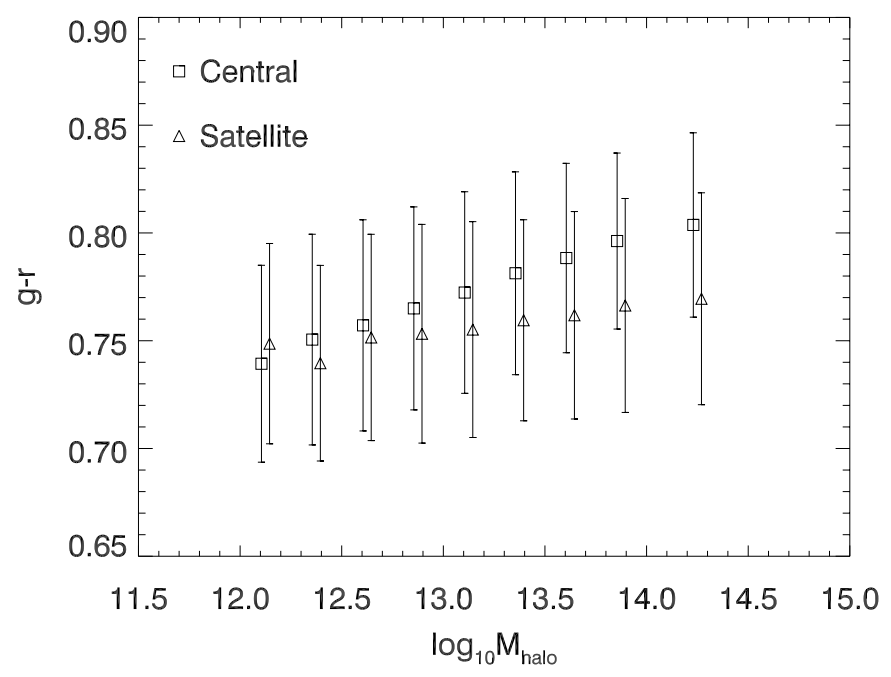

Fig. 1. Dependence of $g-r$ color on the halo mass for central and satellite galaxies. Squares are central galaxies, and triangles are satellite galaxies. Error bars indicate the standard deviation.

\section{Data}

\subsection{Early-type galaxy sample}

ETGs in this work are selected from SDSS DR7 ${ }^{1}$ using similar criteria to Bernardi et al. (2003b):

(1) Concentration parameter $r_{90} / r_{50}>2.5$ in $i$ band;

(2) Ratio of the likelihood of the de Vaucouleurs model to the exponential model $L_{\mathrm{deV}} / L_{\mathrm{exp}} \geq 1.03$;

(3) Spectral classification index eClass $<-0.1$;

(4) Warning flag $z$ Warning $=0$;

(5) $S / N>10$;

(6) Redshift $z<0.2$;

(7) Central velocity dispersion $\sigma>70 \mathrm{~km} \mathrm{~s}^{-1}$.

The sample consists of 70793 galaxies.

\subsection{SDSS group catalog}

The selection procedure and the properties of the group cata$\log$ are described in Yang et al. (2007). A modified version of the halo-based group finder that was developed by Yang et al. (2005) is used to select galaxy groups from the SDSS. In this work, the catalog version based on the SDSS DR7 is used. By cross-correlating the ETG sample with the group catalog, we get the mass of the host dark matter halo for each ETG. We also distinguish each ETG as a central or satellite galaxy of the halo. In this work, we adopt the halo mass and whether a central or satellite galaxy to describe the environment of an ETG. The unit of the halo mass is $h^{-1} M_{\odot}$.

\subsection{Parameters}

We compute the effective angular radius as

$r_{0}=\sqrt{b / a} r_{\mathrm{deV}}$

where $b / a$ is the axis ratio and $r_{\mathrm{deV}}$ is the de Vaucouleurs angular radius. Next, we convert $r_{0}$ into the effective physical radius $R_{0}$ at the corresponding redshift $z$.

1 http://www.sdss.org/dr7/ 
Table 1. $\mathrm{FP}_{*}$ coefficients of the ETG sample.

\begin{tabular}{lccccc}
\hline \hline Band & $a$ & $b$ & $c$ & Scatter $_{\text {orth }}$ & Scatter $_{R_{0}}$ \\
\hline$g$ & $1.511 \pm 0.004$ & $-0.716 \pm 0.001$ & $3.32 \pm 0.01$ & 0.052 & 0.102 \\
$r$ & $1.503 \pm 0.004$ & $-0.751 \pm 0.001$ & $3.65 \pm 0.02$ & 0.051 & 0.099 \\
$i$ & $1.508 \pm 0.004$ & $-0.766 \pm 0.001$ & $3.78 \pm 0.02$ & 0.050 & 0.098 \\
$z$ & $1.479 \pm 0.004$ & $-0.799 \pm 0.002$ & $4.14 \pm 0.02$ & 0.049 & 0.095 \\
\hline
\end{tabular}

Notes. $\mathrm{FP}_{*}$ coefficients of the ETG sample in $g, r, i$, and $z$ bands. Columns 2 to 4 are coefficients $a$, $b$, and $c$. Columns 5 and 6 are scatters in the orthogonal direction and in the $R_{0}$ direction.

Because the SDSS spectrum is observed using a fixed fiber aperture, the velocity dispersion $\sigma$ should be corrected as

$\sigma_{0}=\sigma\left(\frac{r_{\text {fiber }}}{r_{0} / 8}\right)^{0.04}$,

where $r_{\text {fiber }}$ is the angular radius of the fiber, and $r_{0}$ is the effective angular radius (Jorgensen et al. 1995; Wegner et al. 1999).

To fit the $\mathrm{FP}_{*}$ of ETGs, we should calculate the stellar mass $M_{*}$ of each galaxy. In the SDSS group catalog, $M_{*}$ is estimated based on the relation between the stellar mass-to-light ratio and the $g-r$ color (Bell et al. 2003):

$\log M_{*} / L_{r}=1.097(g-r)-0.306$,

where the magnitudes are K-corrected and evolution-corrected to $z=0.0$. Therefore, $M_{*}$ is estimated as

$\log \frac{M_{*}}{h^{-2} M_{\odot}}=-0.306+1.097(g-r)-0.1-0.4\left(M_{r}-5 \log h-4.64\right)$,

where 4.64 is the $r$-band $\mathrm{AB}$ magnitude of the Sun, and -0.1 means that the Kroupa (2001) initial mass function (IMF) is adopted (Borch et al. 2006). The stellar mass surface density is computed as $\Sigma_{0}=M_{*} /\left(2 \pi R_{0}^{2}\right)$. In Sect. 6.2, we will discuss the effect of the IMF by changing the Kroupa IMF to another universal IMF, and to the non-universal IMF.

\section{Fitting methods}

In this work, we use the orthogonal fitting method to calculate coefficients of the $\mathrm{FP}_{*}$. This fitting method is similar to that in Hou \& Wang (2015), except that $I_{0}$ is replaced with $\Sigma_{0}$. Firstly, we calculate the covariance matrix of $\log R_{0}, \log \sigma_{0}$, and $\log \Sigma_{0}$. Then we estimate the corresponding error matrix, and subtract it from the covariance matrix to get the intrinsic covariance matrix. When calculating the error matrix, we assume that the error of stellar mass is not correlated to the error of effective radius and velocity dispersion. By diagonalizing the intrinsic covariance matrix, we obtain the slopes of $\mathrm{FP}_{*}$. The offset of $\mathrm{FP}_{*}$ is calculated by $c=\overline{\log R_{0}}-a \overline{\log \sigma_{0}}-b \overline{\log \Sigma_{0}}$. We use 100 repeated bootstrap samples to estimate the uncertainties of $a, b$, and $c$.

With this method, we measure $\mathrm{FP}_{*}$ coefficients of the ETG sample. Results are shown in Table 1. We find that, compared to the FP (Table 1 of Hou \& Wang 2015), coefficient $a$ of $\mathrm{FP}_{*}$ is closer to 2, but coefficient $b$ of $\mathrm{FP}_{*}$ is similar to that of the FP. This implies that the stellar population can only explain a part of the FP tilt. Therefore, $M_{\text {dyn }} / M_{*}$ is not constant.

\section{Environmental dependence of the $\mathrm{FP}_{*}$}

Hou \& Wang (2015) studied the environmental dependence of the FP, and found that there is a significant difference between central and satellite galaxies. FP coefficients of central galaxies depend strongly on the halo mass but, for satellite galaxies, the dependence on the halo mass is weak. In this work, we study the environmental dependence on the $\mathrm{FP}_{*}$. To do this, we divide the ETG sample into nine halo mass bins (as per Table 2 of Hou \& Wang 2015), and fit the $\mathrm{FP}_{*}$ for each subsample. The $\mathrm{FP}_{*}$ coefficients, as functions of the halo mass, are shown in Fig. 2. We compare the environmental dependence of the $\mathrm{FP}_{*}$ and the FP in Tables A.1 and A. 2 for central and satellite galaxies respectively.

We find the following results. Firstly, in the same halo bin, coefficient $a$ of $\mathrm{FP}_{*}$ is systematically larger than that of the FP, except for in $z$ band. This means that the tilt of the $\mathrm{FP}_{*}$ is indeed mitigated relative to that of the FP. Secondly, coefficient $b$ of the $\mathrm{FP}_{*}$ is similar to that of the FP. Finally, the environmental dependence of the $\mathrm{FP}_{*}$ is similar to that of the FP. Although $\mathrm{FP}_{*}$ coefficients of central galaxies are correlated to the halo mass, those of satellite galaxies are similar in different halo mass bins. These results suggest that the FP tilt is not primarily driven by the stellar population. Moreover, the discrepancy between central and satellite galaxies is not due to the difference of the stellar population in them.

To test whether our results are due to the pollution of latetype galaxies in the sample, we select ETGs that are redder than the dividing line $g-r=0.68-0.030\left(M_{r}+21\right)$, and calculate the environmental dependence of the $\mathrm{FP}_{*}$ of these galaxies. Results are shown in Fig. 3. We find that the pollution of blue galaxies does not change our conclusions.

The FP was considered as a universal scaling relation, in the sense that ETGs with different properties obey a similar FP. However, there is evidence that the scaling relation is curved when expanding the range of galaxy properties (Zaritsky et al. 2006; Wolf et al. 2010; Tollerud et al. 2011). Therefore, when comparing FP coefficients of two samples, we should ensure that galaxies in these samples are located in the same volume of the parameter space. To do this, we fit the distribution of $\log R_{0}$, $\log \sigma_{0}$, and $\log M_{*}$ in each halo mass bin to the double Gaussian. Assuming the fitted peaks and standard deviations of $\log R_{0}$ distribution are $\mu_{1}, \sigma_{1}, \mu_{2}$, and $\sigma_{2}$, with $\mu_{1}<\mu_{2}$, we only keep the galaxies with $\log R_{0}$ in the range $\left[\mu_{1}-\sigma_{1}, \mu_{2}+\sigma_{2}\right]$. Similarly, galaxies are screened so that central and satellite galaxies are in the same range of $\log \sigma_{0}$ and $\log M_{*}$. Afterwards, the remaining galaxies in the same halo mass bin are located in the same volume of $\left(R_{0}, \sigma_{0}\right.$ and $\left.M_{*}\right)$ space. We plot the environmental dependence of the $\mathrm{FP}_{*}$ of the resulting samples in Fig. 4. Here we set the size of the halo mass bin as 0.5 , so that galaxy numbers in all halo mass bins are statistically large enough. We find that the environmental dependence of the $\mathrm{FP}_{*}$ still exists. Therefore, the 

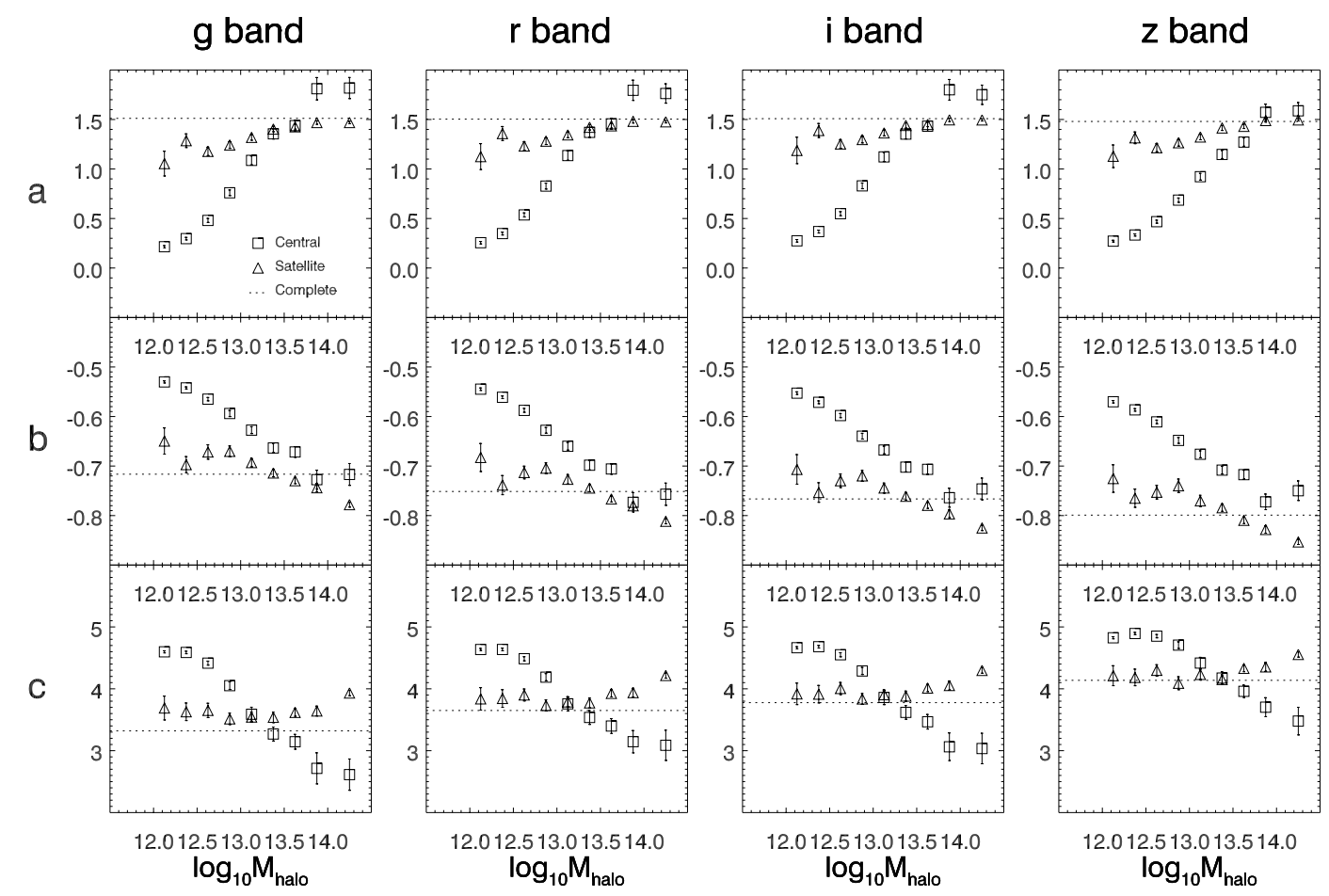

Fig. 2. Environmental dependence of the $\mathrm{FP}_{*}$ in $g, r, i$ and $z$ bands. Squares are central galaxies, and triangles are satellite galaxies. The horizontal dotted lines are the corresponding coefficients of the complete ETG sample.
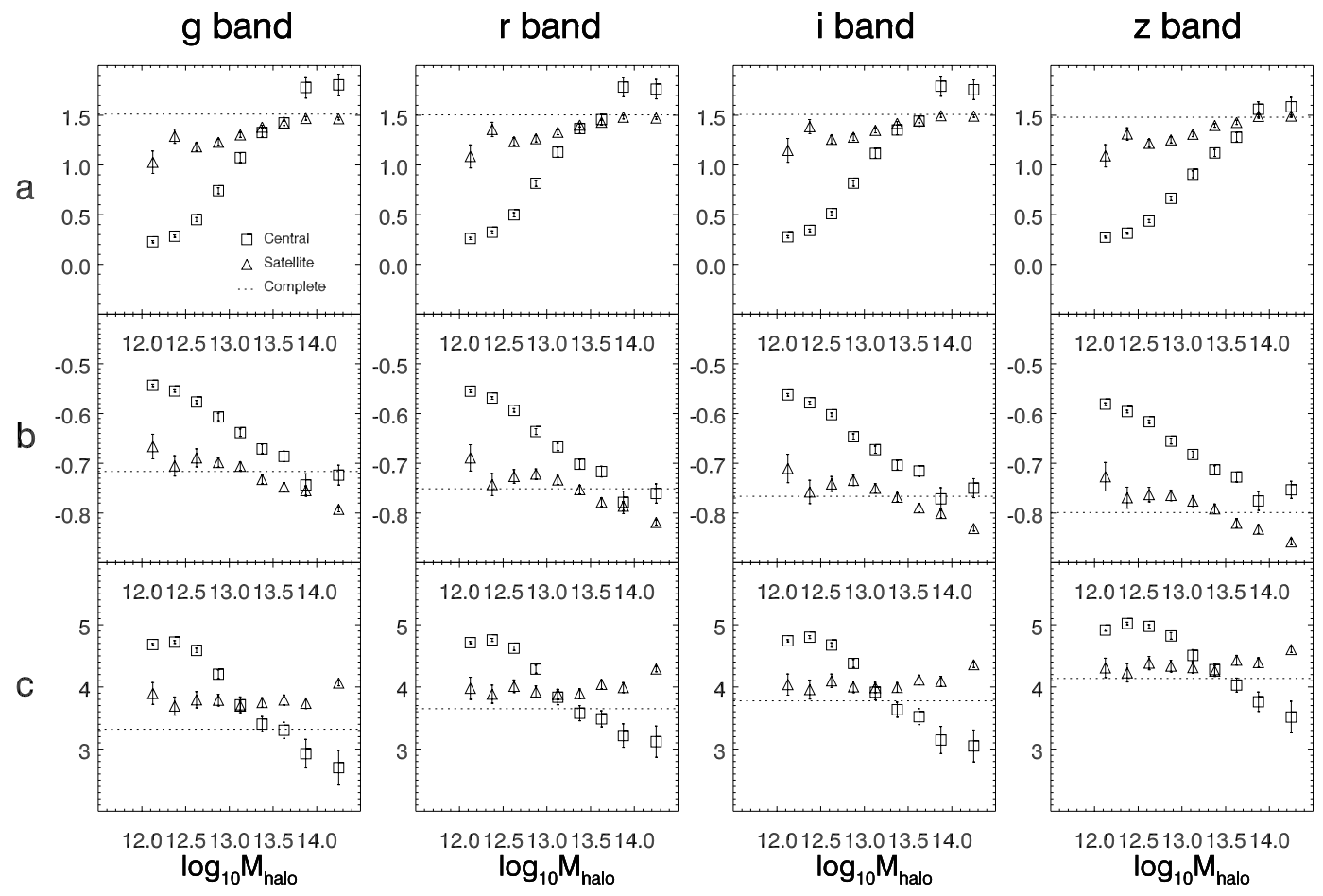

Fig. 3. As in Fig. 2, but galaxies are redder than the dividing line $g-r=0.68-0.030\left(M_{r}+21\right)$.

difference between central and satellite galaxies is not primarily due to the curvature of the scaling relation.

\section{Summary and discussion}

\subsection{Summary}

Using a sample of 70793 ETGs from SDSS DR7 and the SDSS group catalog, we investigate the environmental dependence of the $\mathrm{FP}_{*}$. We find that coefficient $a$ of the $\mathrm{FP}_{*}$ is closer to 2 than that of the FP, but coefficient $b$ of the $\mathrm{FP}_{*}$ is similar to that of the FP. The environmental dependence of the $\mathrm{FP}_{*}$ is similar to that of the FP. The $\mathrm{FP}_{*}$ coefficients of central galaxies are correlated to the halo mass, but those of satellite galaxies are similar in all halo mass bins. These results suggest that the FP tilt cannot be explained by the stellar population, and that the difference between central and satellite galaxies is not due to the stellar population. 
L. Hou and Y. Wang: Environmental dependence of the stellar mass fundamental plane
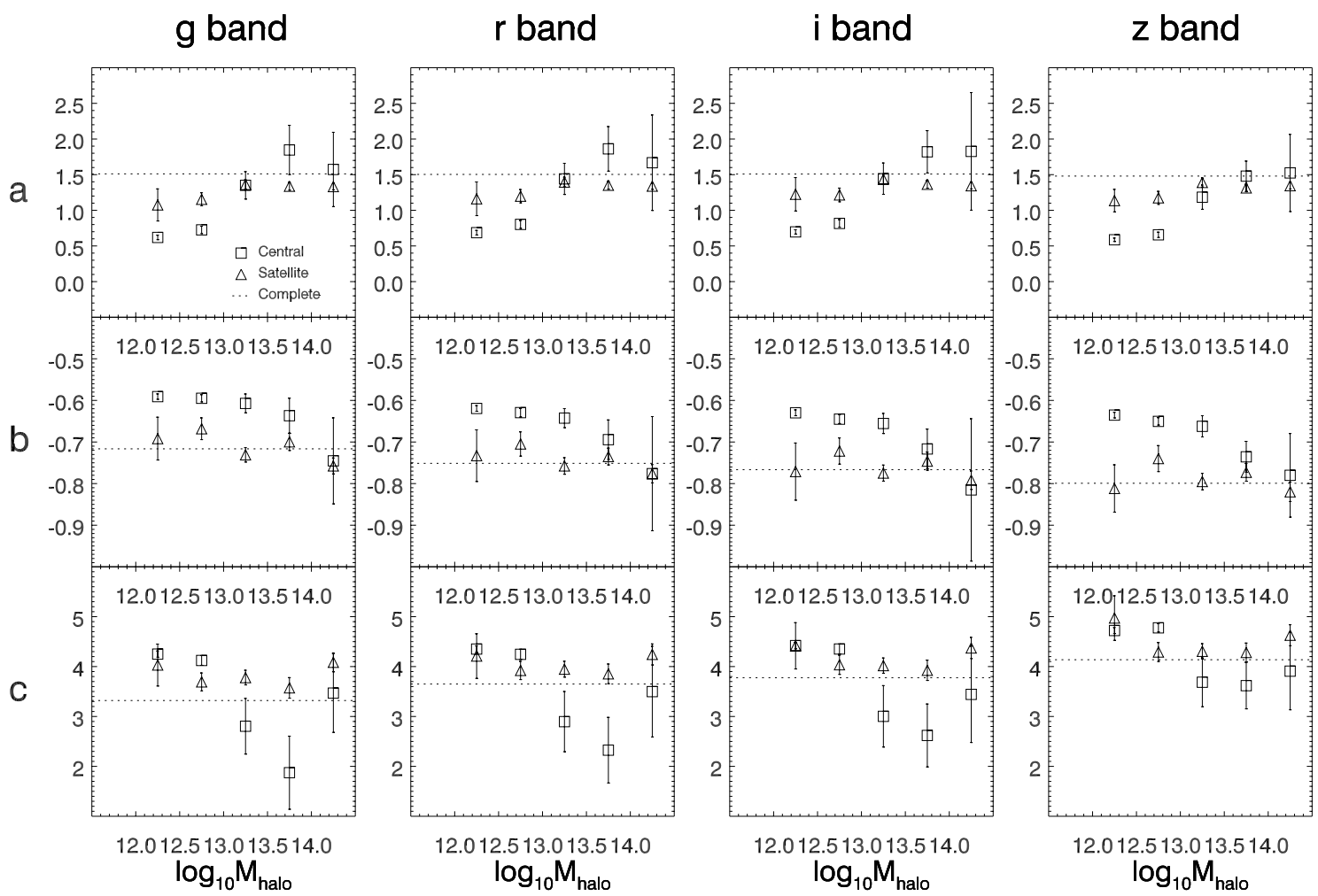

Fig. 4. As in Fig. 2, but galaxies in each halo mass bin are located in the same volume of the parameter space.

\subsection{Explanations of the results}

The similarity between the environmental dependence of the FP and the $\mathrm{FP}_{*}$ implies that the discrepancy between central and satellite galaxies is not due to variations in the stellar mass-tolight ratio $M_{*} / L$. We discuss several explanations for the results as follows.

\subsubsection{Environmental difference between central and satellite galaxies}

$\mathrm{FP}_{*}$ coefficents of central and satellite galaxies reveal different dependencies on the halo mass. One explanation is that central and satellite galaxies are affected by different environmental processes, which make them obey different FPs. There is a significant difference between the properties of central and satellite galaxies (e.g. Weinmann et al. 2006; van den Bosch et al. 2008; Peng et al. 2010, 2012; Moster et al. 2013; Woo et al. 2013; Wetzel et al. 2013). In the standard $\Lambda$ CDM cosmology, the central galaxy resides in the middle of the dark matter halo, where the density is higher and the gravitational potential well is deep, while satellite galaxies are considered as having been previously accreted into the halo and then into the orbit around the central galaxy. Several environmental processes only operate on satellite galaxies, such as ram pressure, tidal stripping, and strangulation. Therefore, the environmental difference between central and satellite galaxies drives them to evolve in different ways. Essentially, this enables the FPs of central and satellite galaxies to be made differently. Furthermore, the concentration of the dark matter halo is smaller in more massive halos (e.g., Navarro et al. 1997; Bullock et al. 2001; Eke et al. 2001; Zhao et al. 2009). As a result, the environmental difference between central and satellite galaxies is more significant in less massive halos. This is consistent with our results: $\mathrm{FP}_{*}$ coefficients of central and satellite galaxies are similar in large halos, but are significantly different in small halos.

An alternative explanation is that the environment does not change the FP, but only affects how galaxies populate the FP. In other words, the FP is driven by a basic physical process, while galaxies in different environments are located on a different portion of the FP. It is difficult to conclude whether this explanation is more relevant, because the connection between the environment and the location on the FP may only be the consequence of the environmental process, mentioned above.

\subsubsection{Rotational component}

Traditionally, different scaling relations have been available to describe the kinematic and photometric properties of disk galaxies (such as the Tully-Fisher relation) and ETGs (such as the Faber-Jackson relation and the FP). In these relations, the kinematics of disk galaxies is described by the rotational velocity $v_{c}$, and that of ETGs is described by the velocity dispertion $\sigma$. However, several studies conclude that the scatter of scaling relations (such as the Tully-Fisher relation and the FP) is smaller by adopting $V \equiv \sqrt{v_{c}^{2} / 2+\sigma^{2}}$ as the kinematic parameter (Burstein et al. 1997; Weiner et al. 2006; Kassin et al. 2007; Zaritsky et al. 2008). Zaritsky et al. (2008) studied galaxies from disks to spheroids and from dwarf spheroidals to brightest cluster galaxies, and found that all galaxies obey the following equation, called the fundamental manifold (FM):

$\log r_{\mathrm{e}}-\log V^{2}+\log I_{\mathrm{e}}+\log \Upsilon_{\mathrm{e}}+0.8=0$,

where $r_{\mathrm{e}}$ is the half-light radius, $I_{\mathrm{e}}$ and $\Upsilon_{\mathrm{e}}$ are the surface brightness and the mass-to-light ratio within $r_{\mathrm{e}}$ respectively. $\Upsilon_{\mathrm{e}}$ includes the contribution of the stellar-to-baryonic mass ratio and the concentration of stars in the halo. Therefore, the scaling relation between the size, kinematics, and luminosity is explained completely by the virial theorem and the behavior of $\Upsilon_{e}$. 

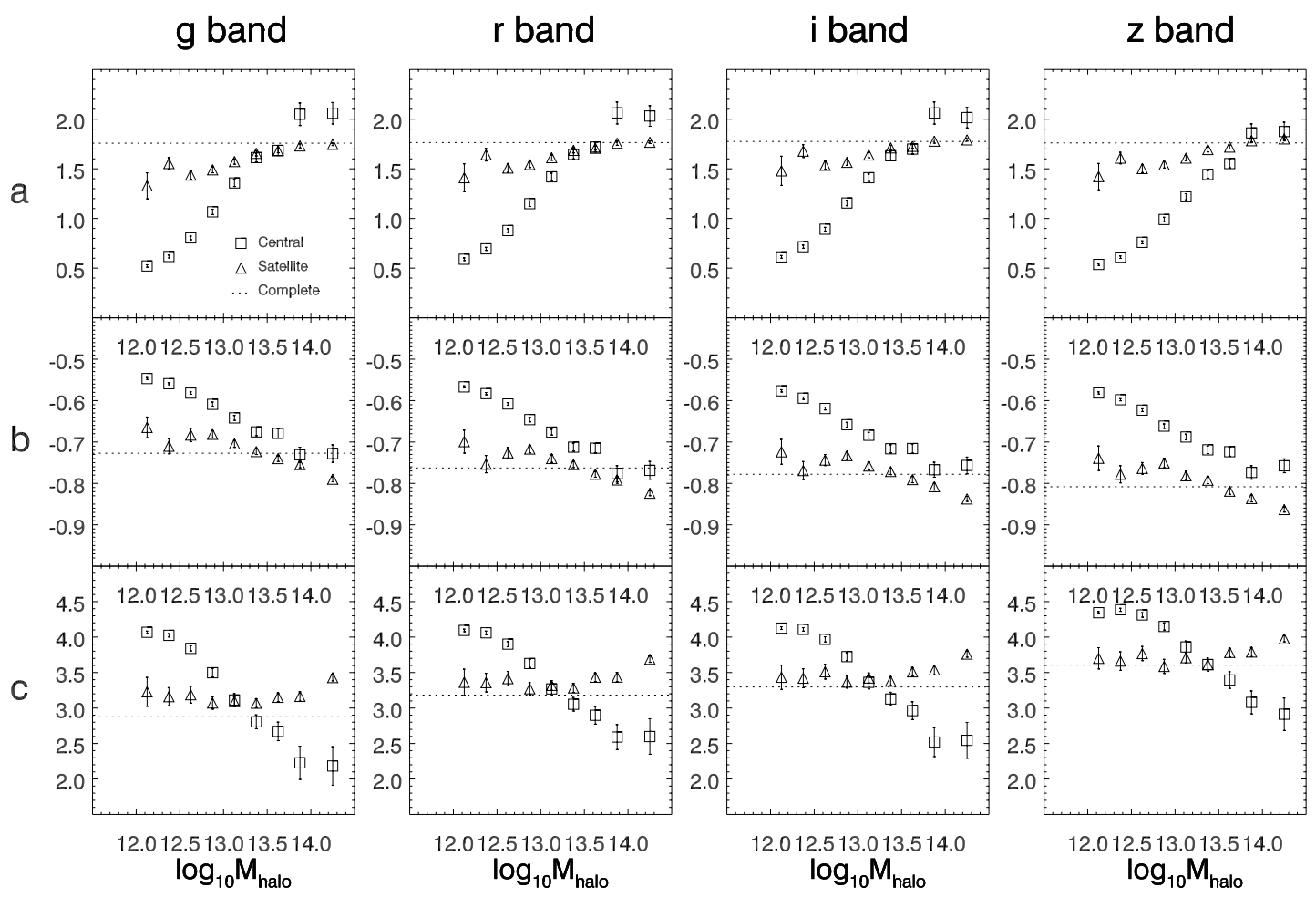

Fig. 5. As in Fig. 2, but the stellar mass $M_{*}$ is corrected to take account of the IMF variations. The correction is based on the relation between the stellar mass and the velocity dispersion, which is parameterized by Cappellari et al. (2013a).

When deriving Eqs. (4) and (6), we assume $V$ is proportional to $\sigma$. This is challenged by the fact that $v_{c} / \sigma$ depends on galaxy properties, such as the luminosity, the color and the isophotal shape of the surface brightness (e.g., Davies et al. 1983; Kormendy \& Bender 1996). There is evidence that satellite galaxies have systematically higher rotational velocity than central galaxies (Desmond \& Wechsler 2015). This may also be responsible for the environmental dependence of the color, because bluer galaxies are likely to have a larger rotational component. However, the SDSS data do not provide the data for rotational velocity. Assuming $V \propto \sigma^{\gamma}$, the existence of the rotational component would make $\gamma<1$, and lead to FP coefficient $a<2$. If $\gamma$ of satellite galaxies is systematically larger than that of central galaxies, coefficent $a$ would be larger for satellite galaxies. Therefore, the environmental dependence of the FP may be due to the missing rotation component in the formulation.

\subsubsection{Differences in the parameter volume}

In Sect. 3, we keep central and satellite galaxies located in the same parameter volume, and find that this correction would not change our conclusions. However, a complete correction should consider the fact that central and satellite galaxies are located in different parameter volumes, which is still an environmental result. The study on central and satellite galaxies, with similar ditributions, would help to solve this problem, but a much larger sample would be required.

\subsubsection{Variations in the IMF}

When calculating the stellar mass, we assume that all ETGs have a Kroupa IMF. Bell \& de Jong (2001) find that, if the IMF is universal, there is a linear relation between the stellar mass-tolight ratio in the band $\lambda$ and the color

$\log \left(M_{*} / L\right)=a_{\lambda}+b_{\lambda} \times$ color.
The slope $b_{\lambda}$ is insensitive to the assumed IMF, but the normalization $a_{\lambda}$ is strongly affected by the IMF. Therefore, changing the Kroupa IMF to another universal IMF produces a constant offset to the estimation of $\log M_{*}$, and thus only produes a constant offset to the coefficient $c$.

However, there is evidence that the IMF is not universal but correlated to the properties of ETGs (e.g., Cenarro et al. 2003; Treu et al. 2010; van Dokkum \& Conroy 2010; Cappellari et al. 2012, 2013a; Spiniello et al. 2012; Posacki et al. 2015). This is described as the IMF mismatch parameter $\alpha \equiv M_{\text {stars }} / M_{\text {pop }}$, where $M_{\text {stars }}$ is the stellar mass that has been calculated using the lensing and dynamical model, and $M_{\text {pop }}$ is the stellar mass that has been calculated using the stellar populations synthesis models with the universal IMF. According to Cappellari et al. (2013a), $\alpha$ is correlated to the velocity dispersion as

$\log \alpha=a^{\prime}+b^{\prime} \log \sigma_{0}$.

To test the effect of the IMF on our conclusions, we make a correction to the stellar mass as

$\log M_{* \text {, corrected }}=\log M_{*}+\log \alpha$,

and use the corrected stellar mass to calculate the environmental dependence of the $\mathrm{FP}_{*}$, as illustrated in Fig. 5. We note that, after the correction on the IMF, coefficent $a$ is systematically larger, coefficient $b$ is not affected, and coefficent $c$ is systematically smaller. Therefore, the correction on the IMF decreases the tilt of the $\mathrm{FP}_{*}$, and does not change the environmental dependence.

\subsubsection{Non-homology}

Several works indicate that the structural and dynamical nonhomology plays an important role in the FP tilt (Trujillo et al. 2004; Nipoti et al. 2006; La Barbera et al. 2010a; D’Onofrio et al. 2013). However, the SAURON project studied the dynamical properties of ETGs, and concluded that $M_{\text {tot }} / L$ can explaine 
most of the FP tilt and that the contribution of non-homology is little (Cappellari et al. 2006, 2013b). Bolton et al. (2008) used the gravitational lensing to estimate the total mass, and also concluded that the non-homology is not important. Therefore, the contribution of the non-homology is still unclear.

\subsubsection{Dark matter fraction}

Cappellari et al. (2006) indicate the dark matter fraction of slowrotating galaxies is higher than that of fast-rotating galaxies. Tortora et al. (2009) found that the dark matter fraction depends on the luminosity of galaxies. Moreover, recent observations and simulations indicated the baryonic fraction is correlated to the halo mass (Giodini et al. 2009; Laganá et al. 2011; Lin et al. 2012; Sun 2012; Balaguera-Antolínez \& Porciani 2013; Velliscig et al. 2014). Variations in the dark matter fraction may be responsible for the environmental dependence.

\subsection{The FP as a distance indicator}

The FP provides an estimation of the size of ETGs if the velocity dispersion and surface brightness are known, and thus can be used as a distance indicator. As the extension of the FP, the fundamental manifold (Eq. (11)) is also used as a fiducial for other distance indicators (Zaritsky et al. 2012). However, the environmental dependence of the FP and the $\mathrm{FP}_{*}$ indicates that there is a systematic bias between galaxies in different environments. The effect of the environment should be considered when scaling relations are used to estimate the distance of galaxies. If the environmental dependence of the FP is due to the incomplete correction for differences in parameter volumes, in other words, the results in this work are only the consequence of the curvature of the scaling relation, the distance estimation would not be affected.

Acknowledgements. We are grateful to Xiaohu Yang for providing the SDSS group catalog. W.Y. would like to acknowledge the support of the Fundamental Research Funds for the Central Universities, 973 Program (No. 2015CB857004), the Science Fund for Creative Research Groups of the National Natural Science Foundation of China (Grant NSFC 11421303), and Nature Science Foundation of Anhui Province.

\section{References}

Balaguera-Antolínez, A., \& Porciani, C. 2013, J. Cosmol. Astropart. Phys., 4, 22 Bell, E. F., \& de Jong, R. S. 2001, ApJ, 550, 212

Bell, E. F., McIntosh, D. H., Katz, N., \& Weinberg, M. D. 2003, ApJS, 149, 289 Bernardi, M., Sheth, R. K., Annis, J., et al. 2003a, AJ, 125, 1866 Bernardi, M., Sheth, R. K., Annis, J., et al. 2003b, AJ, 125, 1817

Bernardi, M., Nichol, R. C., Sheth, R. K., Miller, C. J., \& Brinkmann, J. 2006, AJ, 131, 1288

Bolton, A. S., Treu, T., Koopmans, L. V. E., et al. 2008, ApJ, 684, 248

Borch, A., Meisenheimer, K., Bell, E. F., et al. 2006, A\&A, 453, 869

Borriello, A., Salucci, P., \& Danese, L. 2003, MNRAS, 341, 1109

Bullock, J. S., Kolatt, T. S., Sigad, Y., et al. 2001, MNRAS, 321, 559

Burstein, D., Bender, R., Faber, S., \& Nolthenius, R. 1997, AJ, 114, 1365

Busarello, G., Capaccioli, M., Capozziello, S., Longo, G., \& Puddu, E. 1997, A\&A, 320, 415

Cappellari, M., Bacon, R., Bureau, M., et al. 2006, MNRAS, 366, 1126

Cappellari, M., McDermid, R. M., Alatalo, K., et al. 2012, Nature, 484, 485

Cappellari, M., McDermid, R. M., Alatalo, K., et al. 2013a, MNRAS, 432, 1862
Cappellari, M., Scott, N., Alatalo, K., et al. 2013b, MNRAS, 432, 1709 Cenarro, A. J., Gorgas, J., Vazdekis, A., Cardiel, N., \& Peletier, R. F. 2003, MNRAS, 339, L12

Ciotti, L., Lanzoni, B., \& Renzini, A. 1996, MNRAS, 282, 1

Davies, R. L., Efstathiou, G., Fall, S. M., Illingworth, G., \& Schechter, P. L. 1983, ApJ, 266, 41

de Carvalho, R. R., \& Djorgovski, S. 1992, ApJ, 389, L49

Desmond, H., \& Wechsler, R. H. 2015, MNRAS, 454, 322

Djorgovski, S., \& Davis, M. 1987, ApJ, 313, 59

D’Onofrio, M., Fasano, G., Varela, J., et al. 2008, ApJ, 685, 875

D'Onofrio, M., Fasano, G., Moretti, A., et al. 2013, MNRAS, 435, 45

Dressler, A. 1980, ApJ, 236, 351

Dressler, A., Lynden-Bell, D., Burstein, D., et al. 1987, ApJ, 313, 42

Eke, V. R., Navarro, J. F., \& Steinmetz, M. 2001, ApJ, 554, 114

Forbes, D. A., Ponman, T. J., \& Brown, R. J. N. 1998, ApJ, 508, L43

Giodini, S., Pierini, D., Finoguenov, A., et al. 2009, ApJ, 703, 982

Graham, A., \& Colless, M. 1997, MNRAS, 287, 221

Graves, G. J. 2009, in Galaxy Evolution: Emerging Insights and Future Challenges, eds. S. Jogee, I. Marinova, L. Hao, \& G. A. Blanc, ASP Conf. Ser., 419, 96

Hou, L., \& Wang, Y. 2015, RA\&A, 15, 651

Hyde, J. B., \& Bernardi, M. 2009, MNRAS, 396, 1171

Jorgensen, I., Franx, M., \& Kjaergaard, P. 1995, MNRAS, 276, 1341

Kassin, S. A., Weiner, B. J., Faber, S. M., et al. 2007, ApJ, 660, L35

Kormendy, J., \& Bender, R. 1996, ApJ, 464, L119

Kroupa, P. 2001, MNRAS, 322, 231

La Barbera, F., de Carvalho, R. R., de La Rosa, I. G., \& Lopes, P. A. A. 2010a, MNRAS, 408, 1335

La Barbera, F., Lopes, P. A. A., de Carvalho, R. R., de La Rosa, I. G., \& Berlind, A. A. 2010b, MNRAS, 408, 1361

Laganá, T. F., Zhang, Y.-Y., Reiprich, T. H., \& Schneider, P. 2011, ApJ, 743, 13

Lin, Y.-T., Stanford, S. A., Eisenhardt, P. R. M., et al. 2012, ApJ, 745, L3

Lucey, J. R., Bower, R. G., \& Ellis, R. S. 1991, MNRAS, 249, 755

Magoulas, C., Springob, C. M., Colless, M., et al. 2012, MNRAS, 427, 245

Moster, B. P., Naab, T., \& White, S. D. M. 2013, MNRAS, 428, 3121

Navarro, J. F., Frenk, C. S., \& White, S. D. M. 1997, ApJ, 490, 493

Nipoti, C., Londrillo, P., \& Ciotti, L. 2006, MNRAS, 370, 681

Padmanabhan, N., Seljak, U., Strauss, M. A., et al. 2004, New Astron., 9, 329

Peng, Y.-J., Lilly, S. J., Kovač, K., et al. 2010, ApJ, 721, 193

Peng, Y.-J., Lilly, S. J., Renzini, A., \& Carollo, M. 2012, ApJ, 757, 4

Posacki, S., Cappellari, M., Treu, T., Pellegrini, S., \& Ciotti, L. 2015, MNRAS, 446, 493

Prugniel, P., \& Simien, F. 1996, A\&A, 309, 749

Spiniello, C., Trager, S. C., Koopmans, L. V. E., \& Chen, Y. P. 2012, ApJ, 753, L32

Springob, C. M., Magoulas, C., Proctor, R., et al. 2012, MNRAS, 420, 2773

Sun, M. 2012, New J. Phys., 14, 045004

Tollerud, E. J., Bullock, J. S., Graves, G. J., \& Wolf, J. 2011, ApJ, 726, 108

Tortora, C., Napolitano, N. R., Romanowsky, A. J., Capaccioli, M., \& Covone, G. 2009, MNRAS, 396, 1132

Treu, T., Auger, M. W., Koopmans, L. V. E., et al. 2010, ApJ, 709, 1195

Trujillo, I., Burkert, A., \& Bell, E. F. 2004, ApJ, 600, L39

van den Bosch, F. C., Aquino, D., Yang, X., et al. 2008, MNRAS, 387, 79

van Dokkum, P. G., \& Conroy, C. 2010, Nature, 468, 940

Velliscig, M., van Daalen, M. P., Schaye, J., et al. 2014, MNRAS, 442, 2641

Wegner, G., Colless, M., Saglia, R. P., et al. 1999, MNRAS, 305, 259

Weiner, B. J., Willmer, C. N. A., Faber, S. M., et al. 2006, ApJ, 653, 1049

Weinmann, S. M., van den Bosch, F. C., Yang, X., \& Mo, H. J. 2006, MNRAS, 366,2

Wetzel, A. R., Tinker, J. L., Conroy, C., \& van den Bosch, F. C. 2013, MNRAS, 432, 336

Wolf, J., Martinez, G. D., Bullock, J. S., et al. 2010, MNRAS, 406, 1220

Woo, J., Dekel, A., Faber, S. M., et al. 2013, MNRAS, 428, 3306

Yang, X., Mo, H. J., van den Bosch, F. C., \& Jing, Y. P. 2005, MNRAS, 356, 1293

Yang, X., Mo, H. J., van den Bosch, F. C., et al. 2007, ApJ, 671, 153

Zaritsky, D., Gonzalez, A. H., \& Zabludoff, A. I. 2006, ApJ, 638, 725

Zaritsky, D., Zabludoff, A. I., \& Gonzalez, A. H. 2008, ApJ, 682, 68

Zaritsky, D., Zabludoff, A. I., \& Gonzalez, A. H. 2012, ApJ, 748, 15

Zhao, D. H., Jing, Y. P., Mo, H. J., \& Börner, G. 2009, ApJ, 707, 354 
Appendix A: Additional tables

Table A.1. Environmental dependence of the FP and the $\mathrm{FP}_{*}$ of central galaxies.

\begin{tabular}{|c|c|c|c|c|c|c|c|}
\hline \multirow[t]{2}{*}{ Band } & \multirow[t]{2}{*}{$\log _{10} M_{\text {halo }}$} & \multicolumn{3}{|c|}{$\mathrm{FP}_{*}$ coefficients } & \multicolumn{3}{|c|}{ FP coefficients } \\
\hline & & $a$ & $b$ & $c$ & $a$ & $b$ & $c$ \\
\hline \multirow[t]{9}{*}{$g$} & $(12.00,12.25]$ & $0.215 \pm 0.010$ & $-0.530 \pm 0.002$ & $4.60 \pm 0.02$ & $0.075 \pm 0.014$ & $-0.574 \pm 0.003$ & $-4.28 \pm 0.05$ \\
\hline & $(12.25,12.50]$ & $0.297 \pm 0.015$ & $-0.542 \pm 0.002$ & $4.59 \pm 0.03$ & $0.109 \pm 0.020$ & $-0.586 \pm 0.004$ & $-4.38 \pm 0.07$ \\
\hline & $(12.50,12.75]$ & $0.481 \pm 0.021$ & $-0.565 \pm 0.003$ & $4.41 \pm 0.04$ & $0.265 \pm 0.027$ & $-0.600 \pm 0.004$ & $-4.80 \pm 0.09$ \\
\hline & $(12.75,13.00]$ & $0.760 \pm 0.026$ & $-0.594 \pm 0.006$ & $4.05 \pm 0.05$ & $0.642 \pm 0.046$ & $-0.639 \pm 0.006$ & $-5.96 \pm 0.14$ \\
\hline & $(13.00,13.25]$ & $1.087 \pm 0.045$ & $-0.628 \pm 0.006$ & $3.59 \pm 0.11$ & $1.046 \pm 0.061$ & $-0.669 \pm 0.008$ & $-7.14 \pm 0.19$ \\
\hline & $(13.25,13.50]$ & $1.355 \pm 0.048$ & $-0.664 \pm 0.009$ & $3.27 \pm 0.10$ & $1.203 \pm 0.062$ & $-0.685 \pm 0.008$ & $-7.64 \pm 0.19$ \\
\hline & $(13.50,13.75]$ & $1.438 \pm 0.051$ & $-0.672 \pm 0.011$ & $3.14 \pm 0.13$ & $1.215 \pm 0.065$ & $-0.692 \pm 0.010$ & $-7.73 \pm 0.21$ \\
\hline & $(13.75,14.00]$ & $1.811 \pm 0.128$ & $-0.727 \pm 0.020$ & $2.71 \pm 0.30$ & $1.475 \pm 0.083$ & $-0.738 \pm 0.017$ & $-8.73 \pm 0.31$ \\
\hline & $(14.00, \infty)$ & $1.817 \pm 0.108$ & $-0.717 \pm 0.022$ & $2.61 \pm 0.29$ & $1.389 \pm 0.078$ & $-0.731 \pm 0.016$ & $-8.47 \pm 0.26$ \\
\hline \multirow[t]{9}{*}{$r$} & $(12.00,12.25]$ & $0.255 \pm 0.011$ & $-0.545 \pm 0.002$ & $4.63 \pm 0.02$ & $0.132 \pm 0.013$ & $-0.569 \pm 0.003$ & $-4.19 \pm 0.05$ \\
\hline & $(12.25,12.50]$ & $0.348 \pm 0.017$ & $-0.561 \pm 0.003$ & $4.64 \pm 0.03$ & $0.187 \pm 0.015$ & $-0.585 \pm 0.003$ & $-4.37 \pm 0.06$ \\
\hline & $(12.50,12.75]$ & $0.536 \pm 0.022$ & $-0.588 \pm 0.004$ & $4.49 \pm 0.04$ & $0.340 \pm 0.022$ & $-0.601 \pm 0.004$ & $-4.79 \pm 0.08$ \\
\hline & $(12.75,13.00]$ & $0.827 \pm 0.027$ & $-0.628 \pm 0.006$ & $4.19 \pm 0.05$ & $0.673 \pm 0.037$ & $-0.641 \pm 0.005$ & $-5.84 \pm 0.12$ \\
\hline & $(13.00,13.25]$ & $1.136 \pm 0.048$ & $-0.660 \pm 0.007$ & $3.76 \pm 0.11$ & $1.013 \pm 0.046$ & $-0.671 \pm 0.007$ & $-6.86 \pm 0.15$ \\
\hline & $(13.25,13.50]$ & $1.370 \pm 0.048$ & $-0.698 \pm 0.009$ & $3.54 \pm 0.10$ & $1.185 \pm 0.053$ & $-0.689 \pm 0.008$ & $-7.40 \pm 0.16$ \\
\hline & $(13.50,13.75]$ & $1.456 \pm 0.051$ & $-0.706 \pm 0.011$ & $3.40 \pm 0.12$ & $1.247 \pm 0.062$ & $-0.699 \pm 0.010$ & $-7.63 \pm 0.19$ \\
\hline & $(13.75,14.00]$ & $1.795 \pm 0.121$ & $-0.773 \pm 0.020$ & $3.15 \pm 0.24$ & $1.499 \pm 0.079$ & $-0.752 \pm$ & $-8.66 \pm 0.30$ \\
\hline & $(14.00, \infty)$ & $1.762 \pm 0.100$ & $-0.757 \pm 0.023$ & $3.09 \pm 0.27$ & $1.420 \pm 0.074$ & $-0.744 \pm 0.016$ & $-8.40 \pm 0.25$ \\
\hline \multirow[t]{9}{*}{$\bar{i}$} & $(12.00,12.25]$ & $0.275 \pm 0.011$ & $-0.553 \pm 0.002$ & $4.66 \pm 0.02$ & $0.198 \pm 0.013$ & $-0.576 \pm 0.003$ & $-4.31 \pm 0.05$ \\
\hline & $(12.25,12.50]$ & $0.368 \pm 0.017$ & $-0.571 \pm 0.003$ & $4.68 \pm 0.03$ & $0.269 \pm 0.014$ & $-0.595 \pm 0.003$ & $-4.55 \pm 0.05$ \\
\hline & $(12.50,12.75]$ & $0.549 \pm 0.022$ & $-0.598 \pm 0.004$ & $4.55 \pm 0.04$ & $0.425 \pm 0.021$ & $-0.612 \pm 0.004$ & $-4.98 \pm 0.07$ \\
\hline & $(12.75,13.00]$ & $0.830 \pm 0.026$ & $-0.640 \pm 0.006$ & $4.29 \pm 0.05$ & $0.732 \pm 0.033$ & $-0.654 \pm 0.005$ & $-5.99 \pm 0.11$ \\
\hline & $(13.00,13.25]$ & $1.122 \pm 0.048$ & $-0.668 \pm 0.007$ & $3.86 \pm 0.11$ & $1.023 \pm 0.040$ & $-0.681 \pm 0.007$ & $-6.86 \pm 0.13$ \\
\hline & $(13.25,13.50]$ & $1.352 \pm 0.047$ & $-0.702 \pm 0.009$ & $3.62 \pm 0.10$ & $1.190 \pm 0.048$ & $-0.701 \pm 0.007$ & $-7.40 \pm 0.15$ \\
\hline & $(13.50,13.75]$ & $1.433 \pm 0.050$ & $-0.707 \pm 0.011$ & $3.47 \pm 0.12$ & $1.266 \pm 0.058$ & $-0.704 \pm 0.010$ & $-7.60 \pm 0.18$ \\
\hline & $(13.75,14.00]$ & $1.800 \pm 0.121$ & $-0.764 \pm 0.020$ & $3.06 \pm 0.26$ & $1.508 \pm 0.076$ & $-0.761 \pm 0.016$ & $-8.64 \pm 0.28$ \\
\hline & $(14.00, \infty)$ & $1.750 \pm 0.101$ & $-0.746 \pm 0.020$ & $3.04 \pm 0.28$ & $1.437 \pm 0.071$ & $-0.747 \pm 0.015$ & $-8.36 \pm 0.24$ \\
\hline \multirow[t]{9}{*}{$z$} & $(12.00,12.25]$ & $0.271 \pm 0.007$ & $-0.570 \pm 0.002$ & $4.82 \pm 0.02$ & $0.253 \pm 0.015$ & $-0.584 \pm 0.003$ & $-4.42 \pm 0.05$ \\
\hline & $(12.25,12.50]$ & $0.334 \pm 0.012$ & $-0.587 \pm 0.002$ & $4.89 \pm 0.02$ & $0.333 \pm 0.016$ & $-0.606 \pm 0.004$ & $-4.70 \pm 0.06$ \\
\hline & $(12.50,12.75]$ & $0.468 \pm 0.016$ & $-0.611 \pm 0.003$ & $4.85 \pm 0.03$ & $0.506 \pm 0.024$ & $-0.627 \pm 0.004$ & $-5.20 \pm 0.08$ \\
\hline & $(12.75,13.00]$ & $0.686 \pm 0.018$ & $-0.648 \pm 0.005$ & $4.71 \pm 0.05$ & $0.821 \pm 0.036$ & $-0.673 \pm 0.006$ & $-6.25 \pm 0.12$ \\
\hline & $(13.00,13.25]$ & $0.922 \pm 0.036$ & $-0.676 \pm 0.006$ & $4.42 \pm 0.09$ & $1.094 \pm 0.043$ & $-0.701 \pm 0.007$ & $-7.09 \pm 0.14$ \\
\hline & $(13.25,13.50]$ & $1.147 \pm 0.037$ & $-0.708 \pm 0.008$ & $4.17 \pm 0.08$ & $1.231 \pm 0.048$ & $-0.712 \pm 0.008$ & $-7.49 \pm 0.15$ \\
\hline & $(13.50,13.75]$ & $1.272 \pm 0.043$ & $-0.717 \pm 0.010$ & $3.96 \pm 0.11$ & $1.309 \pm 0.060$ & $-0.718 \pm 0.011$ & $-7.73 \pm 0.19$ \\
\hline & $(13.75,14.00]$ & $1.572 \pm 0.096$ & $-0.772 \pm 0.015$ & $3.70 \pm 0.21$ & $1.529 \pm 0.075$ & $-0.767 \pm 0.016$ & $-8.63 \pm 0.27$ \\
\hline & $(14.00, \infty)$ & $1.588 \pm 0.091$ & $-0.750 \pm 0.019$ & $3.48 \pm 0.27$ & $1.412 \pm 0.068$ & $-0.749 \pm 0.014$ & $-8.21 \pm 0.23$ \\
\hline
\end{tabular}

Notes. The environmental dependence of the FP and the $\mathrm{FP}_{*}$ of central galaxies in $g, r, i$ and $z$-bands. Column 2 is the logarithmic halo mass, Cols. 3 to 5 are $\mathrm{FP}_{*}$ coefficients, and Cols. 6 to 8 are FP coefficients. 
L. Hou and Y. Wang: Environmental dependence of the stellar mass fundamental plane

Table A.2. Environmental dependence of the $\mathrm{FP}$ and the $\mathrm{FP}_{*}$ of satellite galaxies.

\begin{tabular}{|c|c|c|c|c|c|c|c|}
\hline \multirow[t]{2}{*}{ Band } & \multirow[t]{2}{*}{$\log _{10} M_{\text {halo }}$} & \multicolumn{3}{|c|}{$\mathrm{FP}_{*}$ coefficients } & \multicolumn{3}{|c|}{ FP coefficients } \\
\hline & & $a$ & $b$ & $c$ & $a$ & $b$ & $c$ \\
\hline \multirow[t]{9}{*}{$g$} & $(12.00,12.25]$ & $1.055 \pm 0.137$ & $-0.650 \pm 0.025$ & $3.69 \pm 0.19$ & $0.965 \pm 0.132$ & $-0.697 \pm 0.027$ & $-7.30 \pm 0.46$ \\
\hline & $(12.25,12.50]$ & $1.286 \pm 0.072$ & $-0.697 \pm 0.020$ & $3.63 \pm 0.15$ & $1.185 \pm 0.067$ & $-0.721 \pm 0.019$ & $-7.96 \pm 0.26$ \\
\hline & $(12.50,12.75]$ & $1.178 \pm 0.047$ & $-0.672 \pm 0.016$ & $3.65 \pm 0.14$ & $1.067 \pm 0.048$ & $-0.717 \pm 0.014$ & $-7.67 \pm 0.18$ \\
\hline & $(12.75,13.00]$ & $1.242+0.033$ & $-0.670+0.011$ & $3.51 \pm 0.09$ & $1.160+0.035$ & $-0.717+0.009$ & $-7.85+0.13$ \\
\hline & $(13.00,13.25]$ & $1.319 \pm 0.033$ & $-0.693 \pm 0.008$ & $3.54 \pm 0.08$ & $1.204 \pm 0.027$ & $-0.724 \pm 0.009$ & $-8.01 \pm 0.10$ \\
\hline & $(13.25,13.50$ & $1.403 \pm 0.028$ & $-0.714 \pm 0.008$ & $3.54 \pm 0.09$ & $1.278 \pm 0.026$ & $-0.752 \pm 0.008$ & $-8.40 \pm 0.10$ \\
\hline & $(13.50,13.75]$ & $1.426 \pm 0.019$ & $-0.730 \pm 0.008$ & $3.62 \pm 0.07$ & $1.279 \pm 0.023$ & $-0.767 \pm 0.006$ & $-8.53 \pm 0.08$ \\
\hline & $(13.75,14.00]$ & $1.469 \pm 0.020$ & $-0.744 \pm 0.009$ & $3.64 \pm 0.07$ & $1.297 \pm 0.016$ & $-0.776 \pm 0.007$ & $-8.65 \pm 0.07$ \\
\hline & $(14.00, \infty)$ & $1.469 \pm 0.013$ & $-0.778 \pm 0.006$ & $3.93 \pm 0.05$ & $1.291 \pm 0.011$ & $-0.795 \pm 0.005$ & $-8.79 \pm 0.05$ \\
\hline \multirow[t]{9}{*}{$r$} & $(12.00,12.25]$ & $1.125 \pm 0.143$ & $-0.683 \pm 0.029$ & $3.84 \pm 0.18$ & $1.040 \pm 0.136$ & $-0.707 \pm 0.029$ & $-7.33 \pm 0.48$ \\
\hline & $(12.25,12.50]$ & $1.358 \pm 0.073$ & $-0.738 \pm 0.022$ & $3.84 \pm 0.15$ & $1.261 \pm 0.067$ & $-0.745 \pm 0.020$ & $-8.08 \pm 0.26$ \\
\hline & $(12.50,12.75]$ & $1.232 \pm 0.047$ & $-0.713 \pm 0.013$ & $3.90 \pm 0.11$ & $1.132 \pm 0.046$ & $-0.732 \pm 0.014$ & $-7.69 \pm 0.18$ \\
\hline & $(12.75,13.00]$ & $1.281 \pm 0.033$ & $-0.704 \pm 0.011$ & $3.73 \pm 0.09$ & $1.208 \pm 0.035$ & $-0.729 \pm 0.010$ & $-7.82+0.13$ \\
\hline & $(13.00,13.25]$ & $1.345 \pm 0.033$ & $-0.727 \pm 0.009$ & $3.79 \pm 0.09$ & $1.252 \pm 0.027$ & $-0.739 \pm 0.009$ & $-7.99 \pm 0.10$ \\
\hline & $(13.25,13.50]$ & $1.421 \pm 0.028$ & $-0.744 \pm 0.008$ & $3.77 \pm 0.09$ & $1.324 \pm 0.025$ & $-0.760 \pm 0.008$ & $-8.32 \pm 0.10$ \\
\hline & $(13.50,13.75]$ & $1.436 \pm 0.019$ & $-0.767 \pm 0.008$ & $3.93 \pm 0.07$ & $1.326 \pm 0.022$ & $-0.778 \pm 0.007$ & $-8.47 \pm 0.08$ \\
\hline & $(13.75,14.00]$ & $1.481 \pm 0.019$ & $-0.780 \pm 0.009$ & $3.94 \pm 0.07$ & $1.350 \pm 0.016$ & $-0.790 \pm 0.007$ & $-8.62 \pm 0.07$ \\
\hline & $(14.00, \infty)$ & $1.476 \pm 0.013$ & $-0.812 \pm 0.005$ & $4.22 \pm 0.05$ & $1.343 \pm 0.011$ & $-0.808 \pm 0.005$ & $-8.75 \pm 0.05$ \\
\hline \multirow[t]{9}{*}{$i$} & $(12.00,12.25]$ & $1.188 \pm 0.148$ & $-0.707 \pm 0.031$ & $3.92 \pm 0.17$ & $1.103 \pm 0.138$ & $-0.720 \pm 0.031$ & $-7.46 \pm 0.49$ \\
\hline & $2.25,12.50$ & $1.389 \pm 0.077$ & $-0.753 \pm 0.023$ & $3.91 \pm 0.15$ & $1.303 \pm 0.065$ & $-0.764 \pm 0.020$ & $-8.20 \pm 0.26$ \\
\hline & $(12.50,12.75$ & $1.253 \pm 0.048$ & $-0.730 \pm 0.014$ & $4.01 \pm 0.12$ & $1.169 \pm 0.046$ & $-0.745 \pm 0.014$ & $-7.77 \pm 0.18$ \\
\hline & $(12.75,13.00]$ & $1.298 \pm 0.034$ & $-0.720 \pm 0.011$ & $3.84 \pm 0.09$ & $1.235 \pm 0.034$ & $-0.740 \pm 0.010$ & $-7.86 \pm 0.13$ \\
\hline & $(13.00,13.25]$ & $1.364 \pm 0.033$ & $-0.744 \pm 0.009$ & $3.90 \pm 0.08$ & $1.281 \pm 0.026$ & $-0.756 \pm 0.010$ & $-8.08 \pm 0.10$ \\
\hline & $(13.25,13.50]$ & $1.440 \pm 0.028$ & $-0.761 \pm 0.008$ & $3.88 \pm 0.09$ & $1.352 \pm 0.025$ & $-0.775 \pm 0.008$ & $-8.38 \pm 0.10$ \\
\hline & $(13.50,13.75]$ & $1.450 \pm 0.019$ & $-0.779 \pm 0.008$ & $4.01 \pm 0.08$ & $1.357 \pm 0.022$ & $-0.791 \pm 0.007$ & $-8.52 \pm 0.08$ \\
\hline & $(13.75,14.00]$ & $1.496 \pm 0.019$ & $-0.797 \pm 0.009$ & $4.05 \pm 0.08$ & $1.380 \pm 0.016$ & $-0.805 \pm 0.007$ & $-8.68 \pm 0.08$ \\
\hline & $(14.00, \infty)$ & $1.495 \pm 0.013$ & $-0.825 \pm 0.005$ & $4.30 \pm 0.05$ & $1.375 \pm 0.011$ & $-0.821 \pm 0.005$ & $-8.80 \pm 0.05$ \\
\hline \multirow[t]{9}{*}{$z$} & $(12.00,12.25]$ & $1.129 \pm 0.123$ & $-0.725 \pm 0.028$ & $4.21 \pm 0.16$ & $1.229 \pm 0.142$ & $-0.754 \pm 0.034$ & $-7.89 \pm 0.52$ \\
\hline & $(12.25,12.50]$ & $1.315 \pm 0.067$ & $-0.765 \pm 0.021$ & $4.18 \pm 0.14$ & $1.402 \pm 0.071$ & $-0.790 \pm 0.022$ & $-8.51 \pm 0.28$ \\
\hline & $(12.50,12.75]$ & $1.215 \pm 0.046$ & $-0.753 \pm 0.014$ & $4.30 \pm 0.11$ & $1.250 \pm 0.050$ & $-0.762 \pm 0.016$ & $-7.98 \pm 0.20$ \\
\hline & $(12.75,13.00]$ & $1.265 \pm 0.034$ & $-0.740 \pm 0.014$ & $4.09 \pm 0.11$ & $1.299 \pm 0.035$ & $-0.761 \pm 0.011$ & $-8.06 \pm 0.14$ \\
\hline & $(13.00,13.25]$ & $1.323 \pm 0.031$ & $-0.770 \pm 0.009$ & $4.23 \pm 0.08$ & $1.338 \pm 0.027$ & $-0.778 \pm 0.011$ & $-8.27 \pm 0.12$ \\
\hline & $(13.25,13.50]$ & $1.414 \pm 0.027$ & $-0.784 \pm 0.009$ & $4.15 \pm 0.09$ & $1.412 \pm 0.026$ & $-0.790 \pm 0.009$ & $-8.53 \pm 0.10$ \\
\hline & $(13.50,13.75]$ & $1.431 \pm 0.019$ & $-0.810 \pm 0.008$ & $4.33 \pm 0.07$ & $1.409 \pm 0.022$ & $-0.809 \pm 0.007$ & $-8.67 \pm 0.08$ \\
\hline & $(13.75,14.00]$ & $1.490 \pm 0.019$ & $-0.828 \pm 0.009$ & $4.35 \pm 0.07$ & $1.436 \pm 0.017$ & $-0.827 \pm 0.008$ & $-8.87 \pm 0.08$ \\
\hline & $(14.00, \infty)$ & $1.495 \pm 0.013$ & $-0.853 \pm 0.005$ & $4.55 \pm 0.05$ & $1.430 \pm 0.011$ & $-0.836 \pm 0.005$ & $-8.93 \pm 0.05$ \\
\hline
\end{tabular}

Notes. The environmental dependence of the FP and the $\mathrm{FP}_{*}$ of satellite galaxies in $g, r, i$ and $z$-bands. Columns are similar to Table A.1. 